\title{
Dual-wavelength Digital Holography Microscope for BGA Measurement Using Partial Coherence Sources
}

\author{
Hyungjun Cho, Doocheol Kim, and Younghun $\mathbf{Y u}^{*}$ \\ Department of Physics, Cheju National University, Jeju 690-756, Korea \\ Wonki Jung \\ School of Electrical Engineering, Jeonju University, Jeonju 560-759, Korea \\ Sanghoon Shin \\ AP \& Tec, Suseo-dong, Gangnam-gu, Seoul 135-539, Korea
}

(Received July 11, 2011 : revised September 27, 2011 : accepted September 27, 2011)

\begin{abstract}
Dual-wavelength holography has a better axial range than single-wavelength holography, allowing unambiguous phase imaging. Partial coherence sources reduce coherent noise, resulting in improved reconstructed images. We measured a ball-grid array using dual-wavelength holography with partial coherence sources. This holography method is useful for measurement samples that exhibit coherence noise and have a step height larger than the single wavelength used in holography.
\end{abstract}

Keywords: Digital holography, Dual-wavelength holography, Error reduction

OCIS codes : (090.0090) Holography; (090.1995) Digital Holography; (090.4220) Multiplex holography

\section{INTRODUCTION}

Holography records the phase modulation of light reflected or transmitted from a projected object onto a photosensitive plate in the form of interference. A reference and an object beam are required, and an interference pattern is generated as a result of the combination of the two beams. Previously, interference patterns were recorded on film plates. However, charge-coupled device (CCD) and complementary metal-oxide-semiconductor (CMOS) technology are now widely used to capture images, and the holograms are then reconstructed on computers. Yaroslavskii and other researchers first proposed hologram reconstruction on the basis of numerical values in the 1970s [1-5]. Ounral and Scott used numerical reconstruction to measure the size of a particle after improving the reconstruction algorithm [6]. This method of digital recording and reconstruction of a numerical hologram is known as digital holography [7-10]. Digital holography has many advantages; for example, it does not require any chemical processing because the reconstructed image can be observed easily on a computer monitor, and numerical data can be obtained for three-dimensional (3D) objects [11, 12].

However, the phase information becomes ambiguous and causes phase wrapping when the phase change exceeds $2 \pi$. Dual-wavelength holography has been suggested as a way of overcoming this ambiguity [13-16]. Dual-wavelength holography is a technique that extends profile measurements deeper than single-wave holography. Digital holography usually uses a laser, a high-coherence source. The coherent beams are very sensitive to any defect in the optical paths and are affected by coherence noise, which severely reduces the optical quality of the resulting reconstructed images. To eliminate the coherence noise problem, partial coherence sources are used in digital holography. The partial coherence sources are obtained from filtered light-emitting diode (LED) sources or from a laser source with deliberately decreased coherence. These partial coherence sources improved the reconstructed image and reduced coherence noise in digital holography $[17,18]$.

In this study, we measured a ball-grid array (BGA) using a dual-wavelength digital holography technique. We chose to use partial coherence sources because the surface of the

\footnotetext{
*Corresponding author: yyhyoung@cheju.ac.kr

Color versions of one or more of the figures in this paper are available online.
} 
BGA was rough and its height was larger than the source wavelengths. We also used a reference conjugated hologram $(\mathrm{RCH})$ for a phase-compensated phase map in dual-wavelength digital holography.

\section{MODEL}

\section{Hologram Recording and Reconstruction}

In the hologram recording process, a plane reference wave $(R)$ and a diffusively reflected object wave $(O)$ interfere at the CCD. The hologram intensity is given by

$$
I_{H}(x, y)=|\mathrm{R}|^{2}+|\mathrm{O}|^{2}+R^{*} O+R O^{*},
$$

where $R^{*}$ and $O^{*}$ denote the complex conjugates of the reference and object waves, respectively $[1,2]$. The digital holographic image can be recorded using a black-and-white CCD camera. The digital hologram $I_{H}(k . l)$ is an $N \times N$ array resulting from the two-dimensional (2D) sampling of $I_{H}(x, y)$ by the CCD camera. It is given by

$$
I_{H}(k, l)=I_{H}(x, y) \operatorname{rect}\left(\frac{x}{L}, \frac{y}{L}\right) \times \underset{k=-N / 2 l=-N / 2}{N / 2} \sum_{\sum}^{N / 2} \delta(x-k \Delta x, y-l \Delta y),
$$

where $k$ and $l$ are integers, $L \times L$ denotes the area of the CCD chip, and $\Delta x$ and $\Delta y$ indicate the pixel size of the CCD.

In classical optical holography, the object wave can be reconstructed by illuminating the processed hologram with a plane wave similar to that used in the recording process. Looking through the hologram, one observes a virtual image. If a screen is placed at a distance $d$ behind the hologram, a real image is formed on it. Mathematically, the amplitude and phase distributions in the plane of the real image can be found using the Fresnel-Kirchhoff integral $[1,2]$. If a plane wave illuminates the hologram with an amplitude transmittance of $I_{H}(x, y)$, the Fresnel-Kirchhoff integral yields a complex amplitude, $\Psi(\xi, n)$, in the real image plane

$$
\begin{aligned}
& \Psi(\xi, \eta)=\frac{\exp (i 2 \pi d / \lambda)}{i \lambda d} \exp \left[\frac{i \pi}{d \lambda}\left(\xi^{2}+\eta^{2}\right)\right] \times \iint I_{H}(x, y) \exp \left[\frac{i \pi}{\lambda d}\left(x^{2}+y^{2}\right)\right] \\
& \times \exp \left[\frac{i 2 \pi}{\lambda d}(\xi x+\eta y)\right] d x d y,
\end{aligned}
$$

where $\lambda$ is the wavelength, and $d$ is the reconstruction distance. $(\xi, \eta)$ are the coordinates of reconstruction plane, and pixel sizes in the reconstruction plane are $\triangle \xi=\frac{\lambda d}{N \triangle x}$ and $\triangle \eta=\frac{\lambda d}{N \triangle y}$. From Eq. (3), the Fresnel-Kirchhoff integral can be considered the Fourier transformation of the function $I_{H}(x, y) \exp \left[i \pi\left(x^{2}+y^{2}\right) / \lambda d\right]$ at the spatial frequencies $\xi$ and $\eta$. Because $\Psi(\xi, \eta)$ is an array of complex numbers, one can obtain an amplitude-contrast image using the intensity

$$
I(\xi, \eta)=\operatorname{Re}[\Psi(\xi, \eta)]^{2}+\operatorname{Im}[\Psi(\xi, \eta)]^{2} .
$$

The phase-contrast image is obtained by calculating the argument

$$
\phi(\xi, \eta)=\arctan \left\{\frac{\operatorname{Im}[\Psi(\xi, \eta)]}{\operatorname{Re}[\Psi(\xi, \eta)]}\right\} .
$$

The real 3D information is acquired by phase unwrapping with the phase-contrast image. Usually the lasers are used in DHM and are sensitive to any defect in the optical paths, and are affected by coherent noise. These coherent noises degrade the reconstructed image quality. To eliminate the coherent noise, partial coherent sources are suggested in DHM. The partial coherent sources are obtained by laser and RGG [18, 22].

\section{Dual-wavelength Holography and Phase Aberration Compensation}

Consider two single-wavelength phase maps, $\phi_{1}$ and $\phi_{2}$, with an optical path difference, OPD. The beat wavelength $\lambda_{12}$ for $\lambda_{1}$ and $\lambda_{2}$ is given by $\lambda_{12}=\frac{\lambda_{1} \lambda_{2}}{\left|\lambda_{1}-\lambda_{2}\right|}$,

$$
\begin{aligned}
& \phi_{1}\left(x, y ; \lambda_{1}\right)=\frac{2 \pi}{\lambda_{1}} \bullet O P D\left(x, y, \lambda_{1}\right) \\
& \phi_{2}\left(x, y ; \lambda_{2}\right)=\frac{2 \pi}{\lambda_{2}} \bullet O P D\left(x, y, \lambda_{2}\right) \\
& \phi_{12}=\phi_{1}\left(x, y ; \lambda_{1}\right)-\phi_{2}\left(x, y ; \lambda_{2}\right)=2 \pi O P D\left(\frac{1}{\lambda_{1}}-\frac{1}{\lambda_{2}}\right)=\frac{2 \pi O P D}{\lambda_{12}},
\end{aligned}
$$

The value of $\lambda_{12}$ can be increased by selecting values of $\lambda_{2}$ and $\lambda_{2}$. that are closer to each other. The phase map for $\lambda_{12}$ is obtained by subtracting one single-wavelength phase map from the other. This map is called a "coarse map" [14]. To make a corrected $\phi_{12}$ phase map, the aberrations of each phase map, $\phi_{1}$ and $\phi_{2}$, should be compensated. We use reference wave (R) and object wave (O) in DHM. To compensate the phase aberrations we could introduce another wave $\mathrm{O}_{\mathrm{o}}$, corresponding to the object wave without sample [21]. If there are phase aberrations in reference wave and object wave, we could introduce them in waves like Eq. (7).

$$
\begin{aligned}
& \mathrm{R}(\mathrm{x}, \mathrm{y})=|\mathrm{R}| \exp \left[\mathrm{i}\left(\mathrm{k}_{\mathrm{x}} \mathrm{x}+\mathrm{k}_{\mathrm{y}} \mathrm{y}\right)\right] \exp \left[\mathrm{iW}_{\mathrm{R}}(\mathrm{x}, \mathrm{y})\right] \\
& \mathrm{O}_{\mathrm{o}}(\mathrm{x}, \mathrm{y})=\left|\mathrm{O}_{\mathrm{o}}(\mathrm{x}, \mathrm{y})\right| \exp \left[\mathrm{iW}_{\mathrm{o}_{\mathrm{o}}}(\mathrm{x}, \mathrm{y})\right] \\
& \mathrm{O}(\mathrm{x}, \mathrm{y})=\left|\mathrm{O}_{\mathrm{o}}(\mathrm{x}, \mathrm{y})\right| \exp [\mathrm{i} \phi(\mathrm{x}, \mathrm{y})] \exp \left[\mathrm{iW}_{\mathrm{o}_{\mathrm{o}}}(\mathrm{x}, \mathrm{y})\right]
\end{aligned}
$$

Where $\mathrm{k}_{\mathrm{x}}, \mathrm{k}_{\mathrm{y}}$ define the wave propagation direction and $\mathrm{W}_{\mathrm{R}}, \mathrm{W}_{\mathrm{O}_{o}}$ are the phase difference between perfect reference wave and object wave. The reconstructed wave front is given 
by Eq. (8).

$$
\begin{aligned}
\Psi(\mathrm{m}, \mathrm{n})= & \mathrm{C} \times \operatorname{DFT}^{-1}\left\{\mathrm{DFT}\left[\Gamma^{\mathrm{H}}{ }_{\mathrm{RCH}}(\mathrm{k}, \mathrm{l}) \mathrm{I}_{\mathrm{H}}^{\mathrm{F}}(\mathrm{k}, \mathrm{l})\right]\right. \\
& \left.\exp \left[-\mathrm{i} \pi \mathrm{d}\left(\mathrm{v}_{\mathrm{k}}^{2}+\mathrm{v}_{\mathrm{l}}^{2}\right)\right]\right\} \\
\mathrm{I}_{\mathrm{H}}^{\mathrm{F}}=\mathrm{R}^{*} \mathrm{O}= & |\mathrm{R}||\mathrm{O}| \exp \left[-\mathrm{i}\left(\mathrm{k}_{\mathrm{x}} \mathrm{X}+\mathrm{k}_{\mathrm{y}} \mathrm{y}\right)\right] \\
& \exp \left[\mathrm{i}\left(\phi+\mathrm{W}_{\mathrm{o}_{\mathrm{o}}}-\mathrm{W}_{\mathrm{R}}\right)\right]
\end{aligned}
$$

Here $v_{k}=\frac{k}{N \Delta x}, v_{1}=\frac{1}{N \Delta y}$, and $I_{H}^{F}$ is the filtered hologram, and $\mathrm{W}_{\mathrm{o}_{\mathrm{o}}}-\mathrm{W}_{\mathrm{R}}$ describing the aberration. We could compensate the aberration, $\mathrm{W}_{\mathrm{o}_{\mathrm{o}}}-\mathrm{WR}_{\mathrm{R}}$, by introducing the reference conjugated hologram (RCH). To compensate the aberration we introduce the filtered reference hologram ( $\mathrm{I}_{\downarrow}$ $\left.\mathrm{H}^{\uparrow}(\mathrm{R}, \mathrm{F})=\mathrm{R}^{\uparrow} * \mathrm{O}_{\downarrow}\right)$, which is the blank hologram. We define $\Gamma_{\mathrm{RCH}}^{\mathrm{H}}$ as the conjugate phase of Eq. (8),

$$
\Gamma_{\mathrm{RCH}}^{\mathrm{H}}=\exp \left[\operatorname{iarg}\left(\mathrm{I}_{\mathrm{H}}^{\mathrm{F}, \mathrm{F}^{*}}\right)\right]
$$

The multiplication of $\Gamma_{\mathrm{RCH}}^{\mathrm{H}}$ with filtered hologram results in a suppression of the aberration terms [21],

$$
\Gamma_{\mathrm{RCH}}^{\mathrm{H}} \cdot \mathrm{I}_{\mathrm{H}}^{\mathrm{F}}=|\mathrm{R}||\mathrm{O}| \exp (\mathrm{i} \phi)
$$

Eq. (10) shows that the total aberration can be compensated [19-21].

\section{EXPERIMENTAL RESULTS}

Figure 1 shows a schematic of a dual-wavelength transmission holographic microscope. The basic experimental setup is similar to that of a Michelson-type interferometer. A 668-nm laser diode and 677-nm laser diode were used as the light sources. These coherent beams were focused on the scattering surface of a rotating ground glass (RGG). The RGG was rotated in its plane by a motor. The light

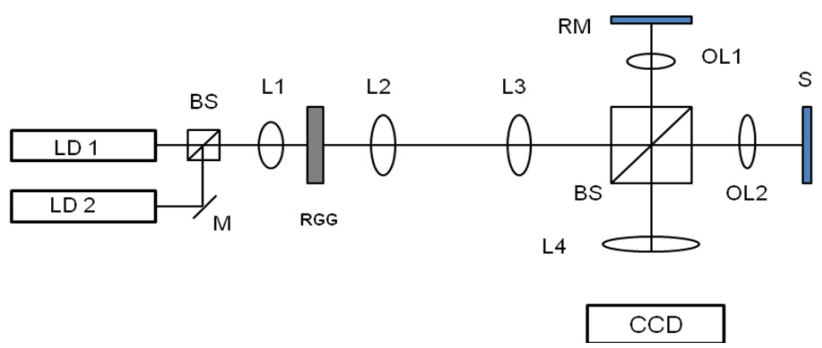

FIG. 1. Schematic experimental set-up for reflection-type dual-wavelength digital holographic microscope. LD 1 and LD 2: laser diodes; BS: beam splitter; M: mirror; RM: reference mirror; L1, L2, L3, and L4: lenses; OL1 and OL2: microscope objective lenses; S: sample; RGG: rotating ground glass; CCD: charge-coupled device. transmitted by the RGG was scattered by its rough surface to create a speckle field that varied with the ground glass rotation. The scattered surface of the RGG was placed in the front focal plane of the lens, L2. Lenses L2 and L3 were used for beam expanding. We used a CCD camera (Sony IPX1M48L) to record the holograms. The pixel size and the number of pixels were $7.4 \mu \mathrm{m} \times 7.4 \mu \mathrm{m}$ and 1024 $\times 1024$, respectively. The CCD was placed in the focal plane of lens 4 (L4). We used a BGA with a height of 15 $\mu \mathrm{m}$ as our sample.

Figure 2 shows the partial coherence effect for the BGA. Figures 2 (a) and (b) present a coherent source image and hologram, (d) and (e) are partial coherence source image and hologram, respectively. Figure 2(c) and (f) are expanded image of dotted square part in Fig. 2(b) and (e). Figures 2 (a) (b) (c) and (d) (e) (f) were obtained without and with the RGG in Fig. 1, respectively. We have used the 50X microscope object lens for the image in Fig. 2 (a) and (d), and Fig. 2(b), (e) are from 20X microscope object lens. The surface of the BGA was rough, which caused coherent noise. We can show that the noise degraded the image and the hologram in Figure 2(a), (c) and the partial coherence source-improved image and hologram in Figure 2(b), (d). The partial-coherence-source method using the RGG resulted in a spatial low-pass filtering in digital holography [18].

Figure 3 shows the dual-wavelength holography experimental results. Figure 3(a) and (d) are the holograms created by laser diode $1(668 \mathrm{~nm})$ and laser diode $2(677 \mathrm{~nm})$, respectively.

Figure 3(b) and (e) show the expanded hologram image of Fig. 3 (a) and (d). Figure 3(c) and (f) show the reconstructed phase map of each hologram. These are not aberration compensated. Each phase map has aberrations, tilt, and centering. We could not extract the right sample phase information without compensating for the tilt aberration. We took the $\mathrm{RCH}$ to compensate for the tilt and centering aberration, and detail process are in Fig. 4 . Figure 4 (a) is

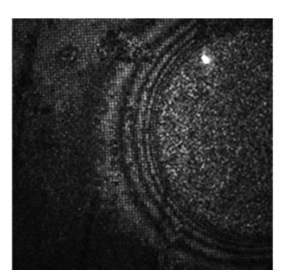

(a)

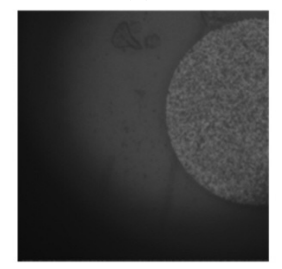

(d)

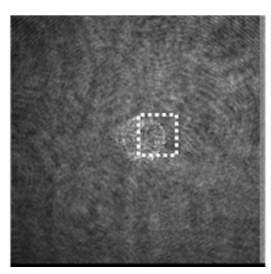

(b)

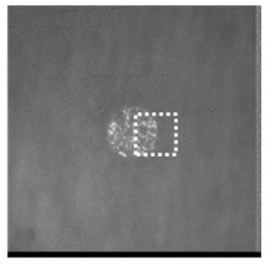

(e)

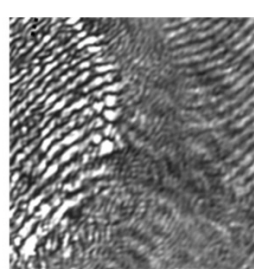

(c)

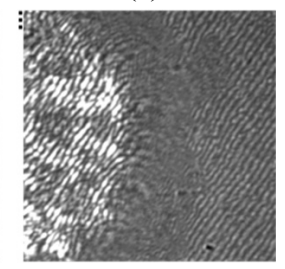

(f)
FIG. 2. Image and holograms created using coherent (a), (b), (c) and partial coherence sources (d), (e), (f). 


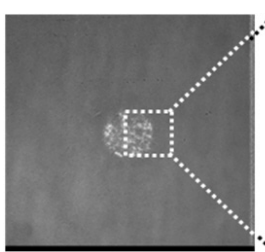

(a)

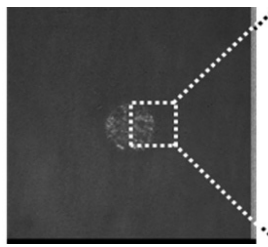

(d)

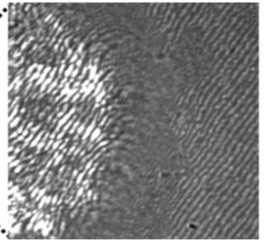

(b)

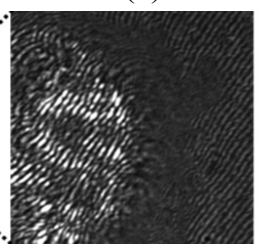

(e)

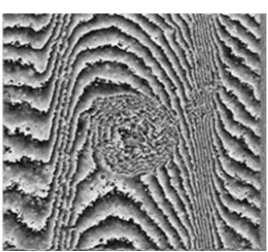

(c)

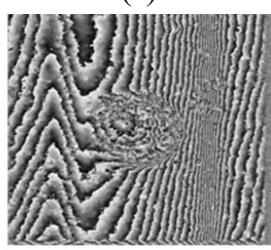

(f)
FIG. 3. Reconstructed BGA phase image. (a), (d) holograms created by the 668-nm source and 677-nm source (respectively); (b),(e) expanded hologram image; (c), (f) reconstructed phase images of (a) and (d) without phase compensation.

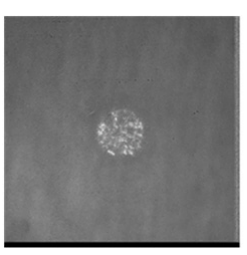

(a)

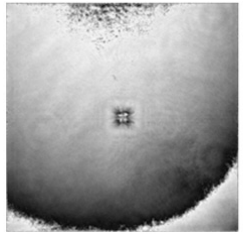

(d)

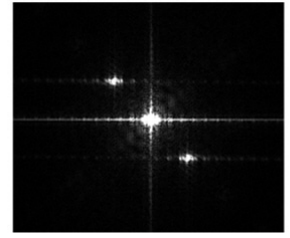

(b)

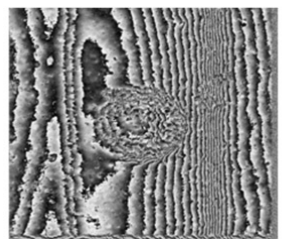

(e)

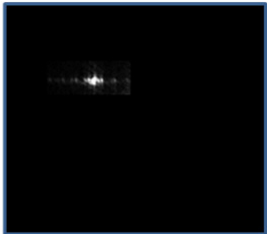

(c)

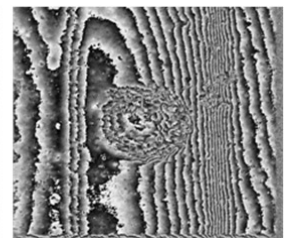

(f)
FIG. 4. Reconstructed BGA phase image and RCH. (a) holograms created by the 668-nm); (b) FFT of (a); (c) filtered hologram; (d) reference; (e),(f) phase aberration compensated phase maps of Fig. 3. (a) and (d).

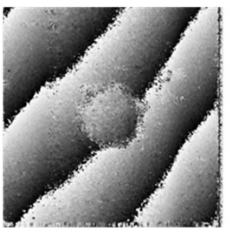

(a)

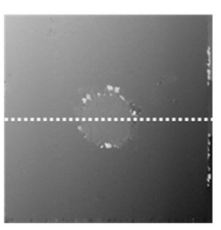

(b)

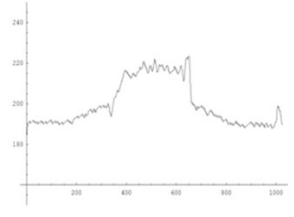

(c)
FIG. 5 (a) reconstructed phase map of equilibrium wavelength; (b) 3D gray image; (c) height measurement.

the hologram, which is same in Fig. 3(a) and Fig. 4(b) is the FFT image of hologram (a). We can get the filtered hologram $\left(\mathrm{I}_{\mathrm{H}}^{\mathrm{F}}\right)$ as like Fig. 4(c) and Fig. 4(d) is the hologram without sample $\left(\mathrm{I}_{\mathrm{H}}^{\mathrm{R}, \mathrm{F}}\right)$. Using Fig. 4(c) and (d) we can get the reconstructed phase map (Fig. 4(e)), which is phase aberration compensated. Figure $4(\mathrm{f})$ is another phase aberration com- pensated phase map using Fig. 3(d). We subtracted phase map Fig. 4(e) from phase map Fig. 4(f) to construct a phase map of the equilibrium wavelength $\left(\lambda_{12}=50248\right.$ $\mathrm{nm})$. The result appears in Figure 5(a). Figure 5(b) shows the gray level of the 3-dimensional in Fig. 5(a) and Fig. $5(\mathrm{c})$ is the height profile from the phase measured along the white line.

We measured the BGA height as $15 \pm 1.2 \mu \mathrm{m}$. The error is relatively large because the error was amplified in the subtraction process between phase maps. This amplification is well known. From these results, we could eliminate the $2 \pi$ ambiguity using dual-wavelength holography and reduce the coherent noise by partial coherence sources. Also, we could use the correct phase information by compensating for the aberration using a $\mathrm{RCH}$.

\section{CONCLUSION}

Dual-wavelength holography is useful for obtaining profile measurements without including a $2 \pi$ ambiguity. Using partial coherence sources reduced coherent noise. The BGA had a rough surface, which caused coherent noise. Its step height was larger than the visible wavelength, which resulted in a $2 \pi$ ambiguity in the digital holography. We used a dualwavelength holography method with partial coherence sources to measure the BGA's height and shape. From experimental results, we showed that the dual-wavelength holography method using partial coherence sources is useful in measurement samples that exhibit coherent noise and whose heights are larger than the single wavelength used in holography.

\section{ACKNOWLEDGMENT}

This work was supported by a research grant from Cheju National University in 2010.

\section{REFERENCES}

1. E. Cuche, P. Marquet, and C. Depeursinge, "Simultaneous amplitude-contrast and quantitative phase-contrast microscopy by numerical reconstruction of Fresnel off-axis holograms," Appl. Opt. 38, 6994-7001 (1999).

2. L. Xu, X. Peng, Z. Guo, J. Miao, and A. Asundi, "Studies of digital microscopy with application to microstructure testing," Appl. Opt. 40, 5046-5051 (2001).

3. M. Jeong, N. Kim, and J. H. Park, "Elemental image synthesis for integral imaging using phase-shifting digital holography," J. Opt. Soc. Korea 12, 275-280 (2008).

4. M. A. Kronrod, N. S. Merzlyakov, and L. P. Yaroslavski, "Reconstruction of hologram with a computer," Sov. Phys. Tech. 17, 434-444 (1972).

5. L. P. Yaroslavskii and N. S. Merzlyakov, Methods of Digital Holography (Consultants Bureau, New York, USA, 1980). 
6. L. Onural and P. D. Scott, "Digital decoding of in-line holograms," Opt. Eng. 26, 1124-1132 (1987).

7. J. W. Goodman, Introduction to Fourier Optics, 2nd ed., J. Goodman, ed. (McGraw Hill, New York, USA, 2005), Chapter 9.

8. U. Schnars and W. Juepther, Digital Holography, U. Schnars and W. Juepther, ed. (Springer, Heidelberg, Germany, 2005).

9. L. Xu, J. Miao, and A. Asundi, "Properties of digital holography based on in-line configuration," Opt. Eng. 39, 32143219 (1999).

10. C. Depeursinge, Digital Holography and Three-dimensional Display, T. C. Poon, ed. (Springer, New York, USA, 2006).

11. D. Kim, B. J. Baek, Y. D. Kim, and B. Javidi, "3D nano object recognition based on phase measurement technique," J. Opt. Soc. Korea 11, 108-112 (2007).

12. S. Shin and Y. Yu, "Three-dimensional information and refractive index measurement using a two-wave digital holographic,” J. Opt. Soc. Korea 10, 173-177 (2006).

13. C. Polhemus, "Two-wavelength interferometry," Appl. Opt. 12, 2071-2074 (1973).

14. J. Gass, A. Dako, and M. K. Kim, "Phase imaging without 2pi ambiguity by multiwavelength digital holography," Opt. Lett. 28, 1141-1143 (2003).

15. Y. Fu, G. Pedrini, B. Hennelly, R. Groves, and W. Osten, "Dual-wavelength image-plane digital holography for dynamic measurement," Opt. Laser Technol. 47, 552-557 (2008).
16. K. Creath, Y. Cheng, and J. C. Wyant, "Contouring aspheric surfaces using two-wavelength phase-shifting interferometry," Opt. Acta 32, 1455-1464 (1985).

17. F. Dubois, L. Joannes, and J. C. Legros, "Improved threedimensional imaging with a digital holography microscope with a source of partial spatial coherence," Appl. Opt. 38, 7085-7094 (1999).

18. F. Dubois, M. L. Requena, C. Minetti, O. Monnom, and E. Istasse, "Partial spatial coherence effects in digital holographic microscopy with a laser source," Appl. Opt. 43, 1131-1139 (2004).

19. T. Colomb, E. Cuche, F. Charrière, J. Kühn, N. Aspert, F. Montfort, P. Marquet, and C. Depeursinge, "Automatic procedure for aberration compensation in digital holographic microscopy and applications to specimen shape compensation," Appl. Opt. 45, 851-863 (2006).

20. S. Shin, D. Kim, and Y. Yu, "Study on digital holography with conjugated hologram," Korean J. Opt. Photon. 21, 146150 (2010).

21. T. Colomb, J. Kühn, F. Charrière, and C. Depeursinge, "Total aberrations compensation in digital holographic microscopy with a reference conjugated hologram," Opt. Express 14, 4300-4304 (2006).

22. J. W. Goodman, Statistical Optics J. Goodman, ed. (Wiley, New York, USA, 1985), Chapter 5. 